\title{
New injectable restorative claims to be strong as a rock
}

GC's new G-aenial Universal Injectable composite range is an easy-to-use composite restorative material that claims to be as strong as a rock.

Available in 16 shades in three opacities, G-aenial Universal Injectable composite restorative material offers exceptional strength and wear resistance thanks to its combination of ultra-fine Barium fillers and GC's Full-coverage Silane Coating (FSC) technology.

Suitable for any cavity class without size limitation, it is said to be strong enough to restore the tooth on its own.

G-aenial Universal Injectable also has a unique thixotropic viscosity which is optimised for free-hand build-up of cusps. This means shorter finishing steps are required, saving time, because the anatomy is already created.

In addition, the new syringe design of G-aenial Universal Injectable enables easy extrusion without the mess and inconvenience of uncontrolled flow-out. Whilst the material's viscosity is optimised for easy separation of the material from the tip, the tips themselves feature a long bendable needle for unparalleled access to difficult to reach posterior cavities.

More information can be found by calling GC UK Ltd on 01908218999 , e-mailing info@gcukltd.co.uk or visiting www.gceurope.com.

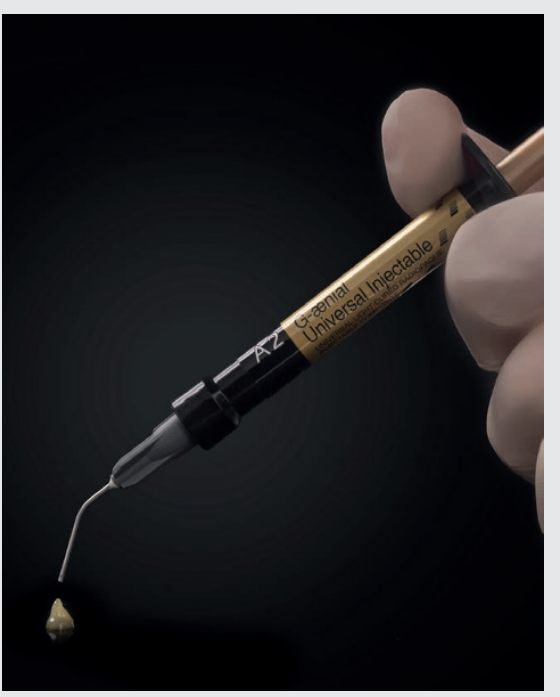

\section{Reliable defence for rugby players}

No matter whether patients play in a professional league or simply enjoy a game on the weekends, rugby can present a number of dangers to their oral and general health.

Dental trauma and concussion rates in the sport are high, so to help protect patients you need to offer a solution that really works.

Give your rugby-playing patients peace of mind by prescribing the Saber Protect mouth guard from CosTech Dental Laboratory.

Made bespoke to the patient's oral cavity, Saber Protect mouth guards are laminated and provide a high level of defence for the teeth, lips, gums and head when players are on the pitch.

The mouth guards have been specially developed to protect players against the impacts they may experience during play and can even help safeguard against concussion due to their innovative design.

They feature varying levels of thickness to best absorb the shock of collisions and tackles and are also made differently depending on which level of rugby patients participate in. This gives patients a truly tailored level of protection that helps significantly lessen the risk of dental injuries.

More information is available at www.costech.co.uk or by calling 01474320076 .

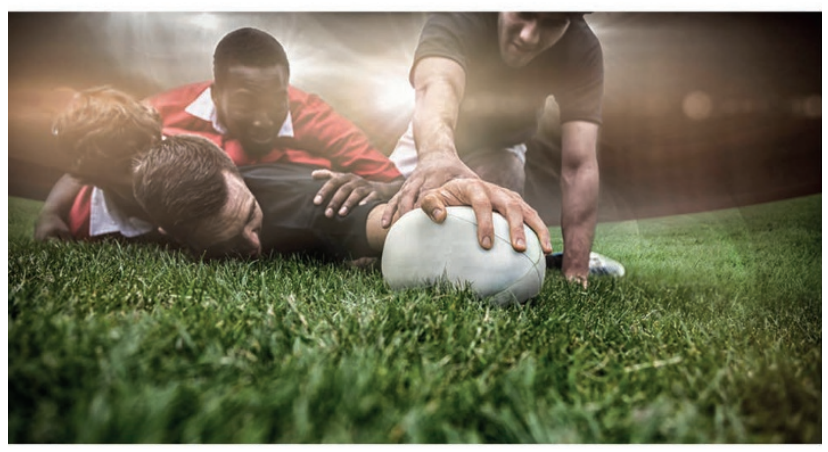

\section{A new world of diagnostic possibilities}

Clark Dental's T-Scan digital occlusal analyser has been praised by Ross Campbell, principal of the Ross Campbell Dental Practice in Norwich.

Dr Campbell says: 'After hearing about the T-Scan from peers, I bought the system from Clark Dental at the end of last year because I was keen to integrate it into my daily practice to help with accurate occlusal analysis and treatment planning.

'My experience of using the T-Scan has been excellent as it easily allows me to balance the occlusion in patients that suffer from TMJ disorders it offers a straightforward way to identify any premature contact.

'The system is well-designed and very easy to use, and my patients find it comfortable during treatment. The graphics allow an easy overview of the occlusion and provide a great patient educational tool. Patients can see what you're doing during treatment, helping them to understand the treatment process and how it will benefit them.

'I would definitely recommend the T-Scan from Clark Dental. It can open up a whole new world of possibilities for dental professionals. I'm very happy with the system, and I'm looking forward to incorporating it more and more into my treatment planning and execution.'

More information is available by calling 01270 613750, emailing info@clarkdentalsales.co.uk or by visiting www.clarkdental.co.uk.

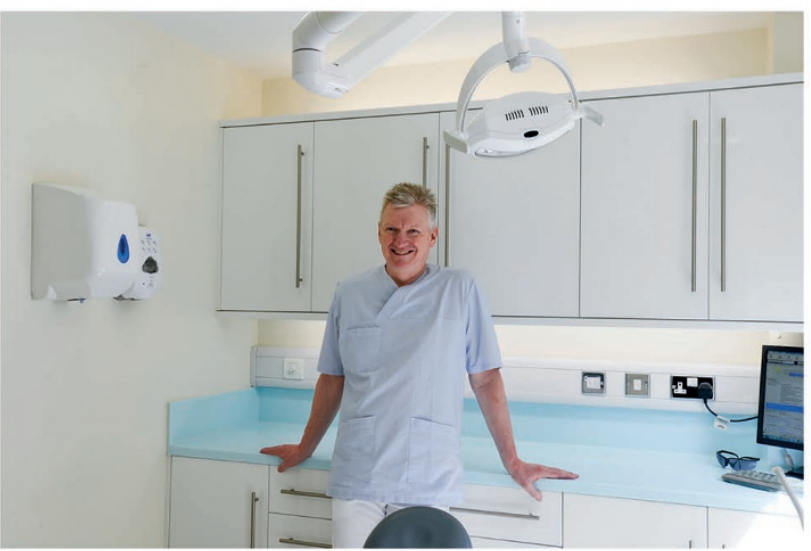

